\title{
A Topology Aggregation Model for Survivability in Multi-Domain Optical Networks Using p-Cycles
}

\author{
Hamza Drid, Samer Lahoud, Bernard Cousin \\ University of Rennes I - IRISA \\ Campus universitaire de Beaulieu 35042 \\ France, Rennes \\ Email: hdrid, slahoud, bcousin@irisa.fr
}

\author{
Miklós Molnár \\ INSA of Rennes - IRISA \\ Dpartement informatique 35043 \\ France, Rennes \\ Email: Molnar@irisa.com
}

\begin{abstract}
Survivability becomes an important issue in optical networks due to the huge bandwidth offered by optical technology. Many works have studied network survivability. The majority of these works are typically destined to singledomain. In this work we address the survivability in multidomain optical networks. This paper provides a classification of the existing protection solutions proposed for multi-domain networks and analyses their advantages and limitations. We propose a new solution for multi-domain optical networks based on p-cycles. For scalability and security reasons, we also propose a topology aggregation model adapted to the p-cycles computation. This aggregation model allows our proposed solution to find a trade-off between two competing goals: efficient use of backup resources and small running time. Simulation results show that the proposed solution is a good trade-off between resource utilization and running time compared to the existing solutions.
\end{abstract}

Keywords-multi-domain; survivability; p-cycle; topology aggregation; optical network;

\section{INTRODUCTION}

Survivability in optical network is an important issue due to the huge bandwidth offered by optical technology. Survivability means that the network has the ability to maintain an acceptable service level even after an occurrence of failures within the network. Therefore operators require incorporating survivability in the design and engineering of their optical networks. This requirement becomes more critical as the size of the networks increases.

Network survivability mechanisms may be classified into two main categories: restoration and protection. The protection is a pro-active approach in which the backup path is reserved at the same time with the working path setup. The restoration is a reactive approach in which a backup path is computed and established after a failure on the working path. The protection is the key mechanism used to ensure survivability in optical networks because it guarantees full recovery whereas the restoration may not offer the same guarantee if resources are not available. Since the singlefiber-link failure is dominant in WDM optical networks, several protection mechanisms against single-link failure have been proposed in the literature. These mechanisms are typically destined to single-domain protection, because they assume that each node in the network has a complete vision of the physical topology of the network. Such an assumption is not realistic in the case of large networks, such as a multidomain networks.

A multi-domain network is a network composed of several single-domain networks, interconnected by inter-domain links. Each domain can be regarded as an independent network that has its own local rules of operation and management to provide services [7]. Due to the scalability constraints and the domain management policies, the internal topological details of a domain are usually not shared externally. As a result, no node in a multi-domain network can have the complete information of a multi-domain network. For instance, complete information corresponds to the detailed states of wavelength usage on each link of a multi-domain optical network. Thus, the protection of multidomain networks is more difficult than that of single-domain networks.

Solutions proposed for the protection problem in multidomain optical networks can generally be classified into two classes. In the first class [1,2,3,4,5], for each connection demand two link-disjoint paths (working and backup path) from source node to destination node are computed. The drawback of this class of solutions is that the recovery time is very long. This is a consequence of the fact that the backup path is routed across the entire network. Moreover, the nodes performing the restoration are the source and destination nodes, and the failures should be notified to all the nodes on the working path. Thus, this protection requires a big number of resource management operations, and it is not acceptable if the domains belong to competitive operators [6].

In the second class, each domain $[6,7,8,10]$ protects the segment of the working path that crosses it. Consequently, the end to end primary light-path is protected. This approach is rather scalable since each network operator protects its domain links independently of the links of the other domains. But the difficulty consists in the protection of the inter-domain links that are not protected by any domain. 
In order to overcome the afore-mentioned drawbacks, we propose to use p-cycles (pre-configured cycles) to protect multi-domain optical networks, and improve the existing solution proposed by A. Farkas and al, in [9]. An important property of p-cycles is that the cycles are fully preconfigured with pre-planned spare capacity and when a link fails, only the two end nodes of the failed link need to perform the recovery actions, and no switching actions are required at any intermediate nodes of the cycles. This property significantly improves the p-cycles recovery time. For scalability and security reasons, we propose a new topology aggregation model to represent each domain in a compact manner. Several topology aggregation models are available in the literature $[12,13,14]$. However, the model that we propose is designed for p-cycle computation.

In the following section, we give an overview about protection using $\mathrm{p}$-cycles. In addition, we present a p-cycle based protection scheme proposed for multi-domain optical networks [9] and its limitation. In sections 3 and 4, we detail our solution and show the performance results. In section 5, we conclude this paper.

\section{Multi-Domain Protection Using P-CyCles}

Single domain network protection based on p-cycles has been extended for multi-domain networks protection. The use of p-cycles for multi-domain networks protection is proposed and evaluated in [9]. In this section of the article, we explain the concept of p-cycles and its advantages. In addition, we describe the solution proposed in [9] and its limitations.

\section{A. p-Cycles}

A p-cycle is an efficient approach for protecting working capacities in WDM networks, because p-cycles can achieve fast restoration time like ring protection and high efficiency capacity like mesh protection [11]. P-cycle protection achieves fast restoration time since only the nodes adjacent to the failure will perform protection switching. The high capacity efficiency is due to the fact that a p-cycle can provide protection not only for on-cycle links but also for straddling links without requiring any additional spare capacity. A straddling link is a link, which does not belong to the p-cycle but whose both end-nodes are on the p-cycle. This property reduces effectively the required protection capacities.

Figure 1 depicts an example that illustrates p-cycle protection. In figure 1 (a), a-b-c-d-e-a is a p-cycle with one unit of spare capacity (i.e. one wavelength) on each on-cycle link. When the on-cycle link (d-c) fails as shown in figure 1 (a), the p-cycle provides one protection path (d-e-a-b-c). In figure 1 (b) an example of a straddling link failure is shown. When the straddling link (e-c) breaks, the p-cycle can protect two working wavelengths on this link by providing respectively two alternate paths (e-d-c) and (e-a-b-c).

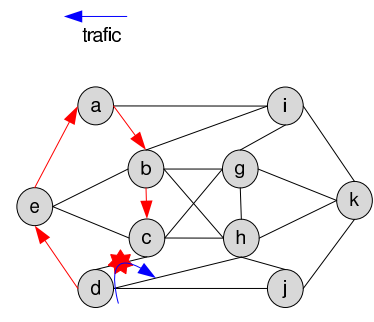

(a)

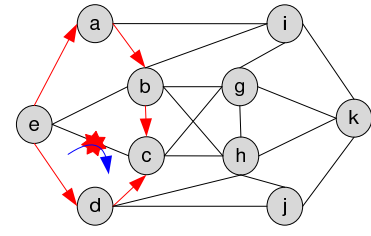

(b)
Figure 1. p-cycle protection

The efficiency of a p-cycle is inversely proportional to its redundancy. The redundancy [15] of a cycle is defined as the ratio between the spare capacity (number of wavelengths used by this cycle) and the working capacity of this cycle (number of wavelengths protected by the cycle on its oncycle and straddling links). A p-cycle with high efficiency is a p-cycle that has a small value of redundancy, i.e. it protects more working wavelengths using few spare wavelengths. We define the redundancy of a p-cycle $i$ as following:

$$
\Re(i)=\sum_{\forall j \in E} P_{i, j} / \sum_{\forall j \in E} X_{i, j}
$$

Let $\mathrm{E}$ be the set of network links. $P_{i, j}$ is the number of working capacity required on link $j$ to construct the p-cycle $i$. $x_{i, j}$ is the number of wavelengths that the p-cycle $i$ protects on the link $j . x_{i, j}$ takes the value 2 if $i$ is a straddling links of p-cycle $i$, and it takes the value 1 if $j$ is an on-cycle link of the p-cycle $i$.

The redundancy $\mathrm{R}$ of the final protection solution is computed as the ratio between the total spare capacity used for protection design and the protected working capacity in the network.

$$
\Re=\sum_{\forall j \in E} S_{j} / \sum_{\forall j \in E} W_{j}
$$

$s_{j}$ and $w_{j}$ are the number of spare and working wavelength capacity on link $j$.

\section{B. P-cycle Based Protection Schemes for Multi- Domain Networks}

In [9], A. Farkas and al, have proposed a solution for a multi-domain networks protection based on p-cycles. The main goal of this solution is to protect the inter-domain links. This solution proceeds in three steps. In the first step, the set of p-cycles protecting the inter-domain links is computed. This set of p-cycles is designed over a singlenode virtual topology (cf. Fig. 2 (b)), in which each domain is represented by a single virtual node. The second step consists of determining for each p-cycle calculated in the previous step, the border nodes to which its links (the on-cycle and straddling links) are connected. The border node connected to the on-cycle inter-domain link of a pcycle is called on-cycle border nodes (denoted by cbn) and 


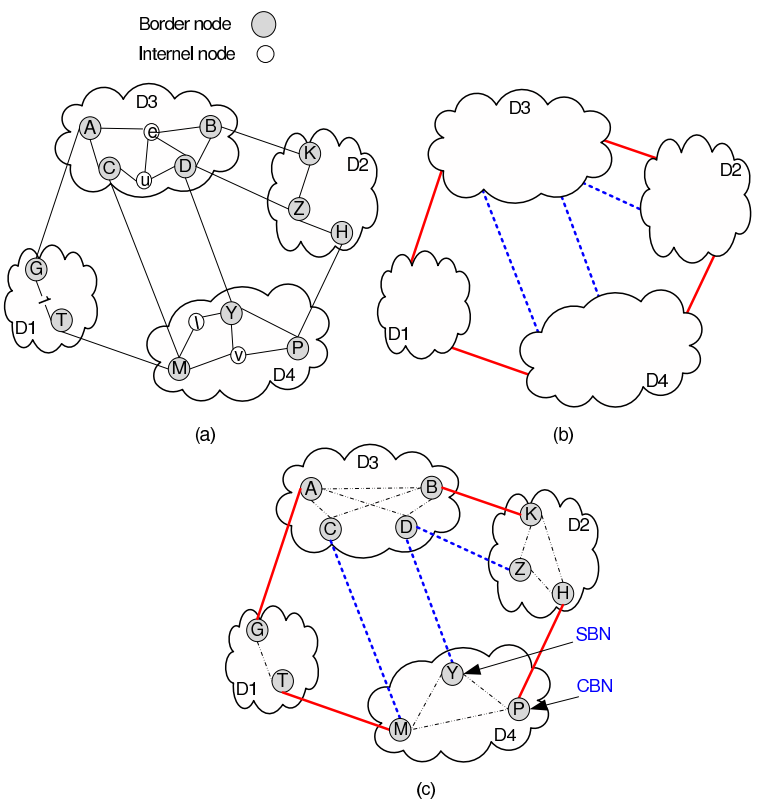

Figure 2. Multi-domain protection using p-cycle

those connected to a straddling inter-domain links are called straddling border nodes (denoted by sbn). Moreover, in this step, the end nodes of inter-domain on-cycle and straddling links need to be connected internally to ensure the continuity of the p-cycle. The internal links connecting the cbn and the sbn nodes are virtual (cf. Fig. 2(c)). During the last step, each internal virtual link calculated in the second step is translated into a physical path. Note that the calculated pcycles bypass the intra-domain links without the possibility to protect them.

This approach presents some drawbacks related to the quality of the set of p-cycles protecting the inter-domain links. At first, the p-cycles protecting the inter-domain links are computed over a single-virtual-node topology. This virtual topology summarizes a domain with multiple links and nodes into a single virtual node. Such representation hides all topological information describing the domain. Consequently, the real cost of a p-cycle on the physical topology is unknown. The second drawback, which we can point out in this solution, is due to the fact that the set of $\mathrm{p}$ cycles obtained is designed only to protect the inter-domain links. Nevertheless, there exists communications within each domain which can be protected by the existing p-cycles, without using additional resources. The communication links which can be protected freely are those passing through the intra-domain links crossed by the existing p-cycles.

\section{Our Proposed Solution}

After presenting the existing solution [9] and its major drawbacks, we develop in this section our motivations and the objectives of the new approach that we propose for multidomain protection using p-cycles. We start by a thorough analysis of the limitations presented in the previous section. Then we introduce the main guidelines of our solution that enables to overcome these limitations.

\section{A. Motivations}

As we have mentioned previously, the solution suggested in [9] presents some disadvantages that strongly affect the quality of the p-cycle set protecting the network (e.g. the redundancy is very high). These disadvantages are mainly due to the lack of topology information of each domain. The following example shows how the lack of topology information of each domain affects the quality of p-cycles during the computation of p-cycles. In this example, we calculate the optimal set of p-cycles (optimality in term of redundancy) on two different topologies of the same multi-domain network (figure 3(a)). In the first topology, all domains (D1 to D4) are represented by their physical topology (i.e. the original topology shown in Figure 3(a)).

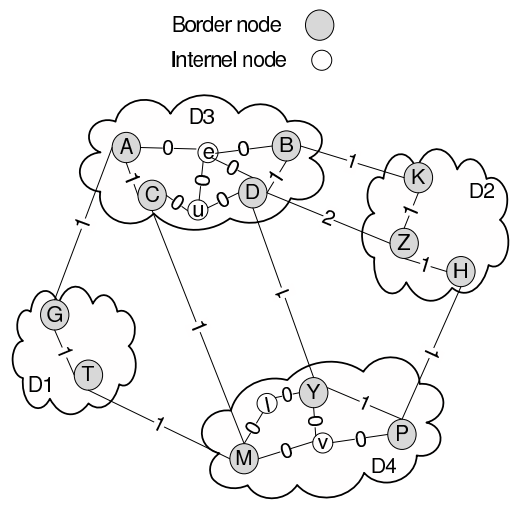

(a)

Figure 3. Multi-domain optical networks

In the second topology, each domain is viewed as a single node as shown in Figure 5 (a). The optimal set of p-cycles obtained for these two topologies are different. In the first topology, the solution consists of two p-cycles (A-C-M-TG-A) and (D-B-K-Z-H-P-Y-D) as shown in figure 4 (a).

In the second topology, the solution is composed of one p-cycle which traverses D1, D2, D3, D4 as shown in figure 5 (a). To evaluate the efficiency, we calculate the redundancy of each solution. The redundancy obtained by the first solution is: $\Re=12 / 14=85.65 \%$.

To calculate the real redundancy of p-cycles computed on the single-virtual-node topology we need to project these p-cycles on the physical topology as shown in figure 5(b). After the projection of p-cycles on the real topology, the redundancy obtained is: $\Re=14 / 14=100 \%$. We note that the redundancy obtained by the first solution is better than that obtained by the latter one. This difference is due to the fact that the first solution takes into account the internal topology of each domain during the computation of p-cycles. 


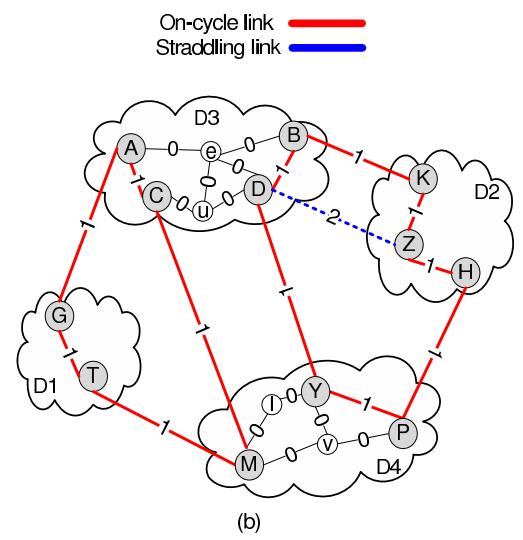

Figure 4. Computed p-cycles with internal topological knowledge

To overcome these problems, the internal topology of each domain should be taken into account during the p-cycles computation. However, as the size of multi-domain network is very large, the computation of the set of p-cycles becomes intractable as soon as we take into account all the details of the internal topology of each domain. Moreover network operator of each domain is very reluctant to distribute the data of their internal network topology. One solution to deal with this scalability problem is to represent the internal topology of each domain in a compact and abstract manner. This process is known as Topology Aggregation.
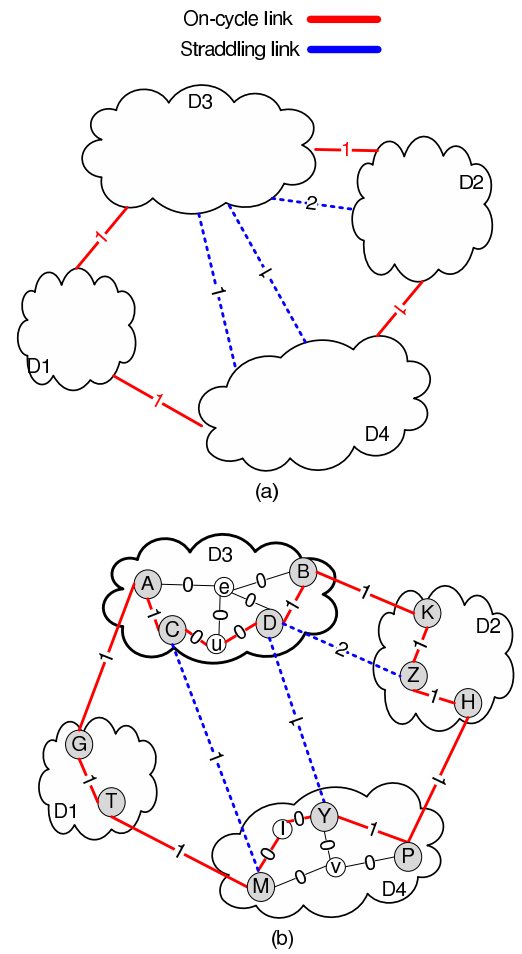

Figure 5. Computed p-cycles with single-virtual-node domains

The basic idea of our proposal consists of describing the internal topology of each domain in a compact manner by using a topology aggregation model. This enables us to construct a new reduced multi-domain topology, on which we calculate the set of p-cycles protecting the network. As a result, the calculation of the p-cycles will be accelerated while the scalability and confidentiality constraints are satisfied. Moreover the quality of the p-cycles will be improved due to following reasons:

1. During the calculation of p-cycles, we have more information about the physical topology of each domain. This solves the first disadvantage of the proposal in [9].

2. Each calculated p-cycle may protect some working capacities into the traversed domains. This overcomes the second limitation of the proposal in [9].

Each calculated p-cycle will contain a set of virtual links inside each of the traversed domain. Each virtual link will be further translated into a light-path.

\section{B. A Novel Topology Aggregation Model}

The aggregation model that we propose in this article aims to represent the network topology and its working capacity in a compact manner. This model is also adapted to the $\mathrm{p}$ cycles computation. We transform the physical topology of each domain into a simple virtual topology.

The topology obtained after aggregation is composed of all border nodes, which are connected by virtual links. The virtual topology has the following characteristics:

- Each pair of border nodes within a domain is connected by a virtual link. A virtual link connecting a pair of border nodes corresponds to the set of the primary lightpaths interconnecting these nodes in the physical topology (see figure $6(\mathrm{c}))$.

- With each virtual link, an integer is associated indicating the number of primary lightpaths existing between the two border nodes.

- Two virtual links have to be physically disjoint, i.e., the lightpaths connecting one pair of border nodes must be link disjoint of all the lightpaths between any other pair of border nodes.

Figure 6 describes an example that shows how our aggregation model generates a virtual topology. Figure 6 (c) describes a network with six nodes, of which three (A, E, F) are border nodes. On each link, an integer value indicating the working capacity of the link is associated. This working capacity is obtained by routing the demand matrix (e.g. using the shortest paths).

In Figure 6(e) the virtual topology obtained from the original topology by aggregation is shown. The virtual topology is only composed of border nodes and virtual links.

The capacities of the links (A-E), (A-F) and (F-E) in the virtual topology indicate the number of disjoint light-paths existing between each border node pair. The virtual link (AE) corresponds, for instance, to the following light paths: two lightpaths which use the path $(A \longrightarrow c \longrightarrow d \longrightarrow E)$ 


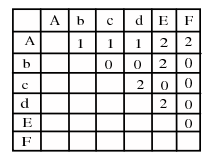

(a) Demands matrix
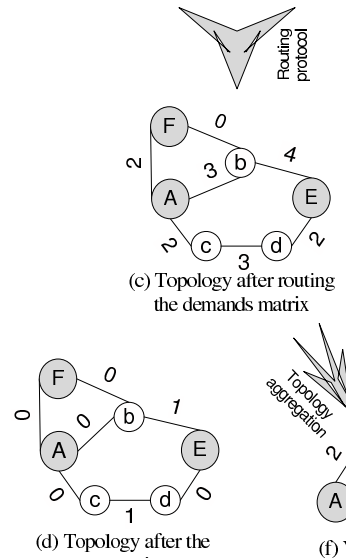

aggregation

Figure 6. Topology aggregation model

and three lightpaths which use $(A \longrightarrow b \longrightarrow E)$. The virtual link A-F corresponds, for instance, to two lightpaths which use the fiber A-F.

We also illustrate in figure 6 (d) the remaining working capacity that could not be aggregated in the virtual topology.

After building the virtual topology of each domain, the set of p-cycles is computed over the virtual topology of the multi-domain network.

Note: The virtual topology of the multi-domain network is composed of the virtual domains, which are interconnected by the inter-domain links.

One of the important properties of a p-cycle is that its links must be physically disjoint. This property ensures that the traffic crossing a potential failing link can be rerouted on the other links of the p-cycle without any interruption of the traffic. For this reason, the virtual links of the topology have to be physically disjoint. If the computed virtual topology does not have this property, the failed link and the links that will protect it, might share some physical links. Consequently, when the failure occurs, several virtual links of different p-cycles can fail at the same time and the traffic will not be recovered.

With the help of the following example, we illustrate the aforementioned disjointness constraint. In figure 7 (b), A-KB-D-C is a p-cycle composed of five links and goes through three different domains D1, D2 and D3. When the on-cycle link A-K fails, the p-cycle provides one protection path (AC-D-B-K) to protect one wavelength of the virtual link A-K.

Figures 7 (b) and (c) illustrate two possible virtual topologies that we can obtain from the original topology showed in figure 7 (a).

In the first virtual topology, let us suppose that all virtual

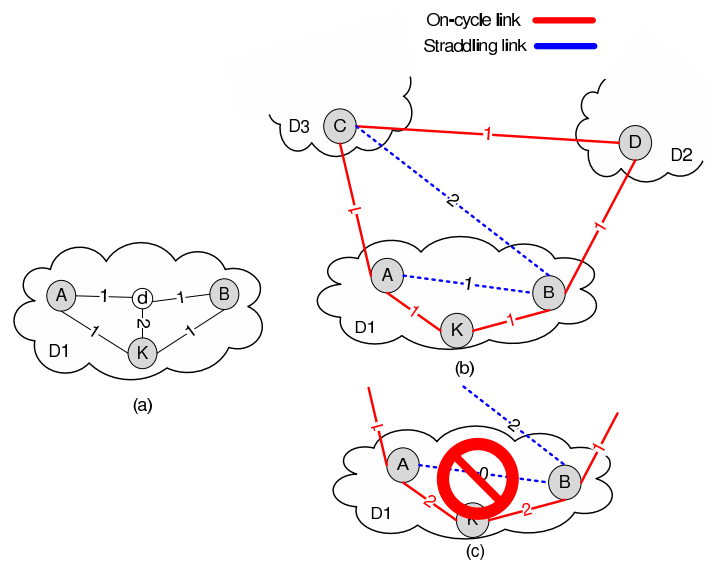

Figure 7. Proposed model of topology aggregation

links are physically disjoint. For instance, the virtual link A$\mathrm{K}$ (K-B, and A-B respectively) corresponds to the lightpath A-K (K-B, A-d-B respectively) in the original topology. In this case, the traffic can be recovered in domain D1.

In the second virtual topology showed in figure 7 (c) the virtual links A-K (which corresponds to the lightpaths A-d$\mathrm{K}$ and $\mathrm{A}-\mathrm{K}$ ) and $\mathrm{K}-\mathrm{B}$ (which corresponds to the lightpath $\mathrm{K}-\mathrm{d}-\mathrm{B}$ and K-B) share the physical link d-K. Consequently, if this link fails the two virtual links fail at the same time and the traffic cannot be rerouted.

\section{ILP Model for Topology Aggregation}

In this section, we present an ILP (Integer Linear Programming) formulation for our topology aggregation. We start by introducing some notations for our model. A multidomain networks composed of $\mathrm{M}$ connected domains can be represented by a graph $G=\left(O_{i}, E\right) \mathrm{i}=1,2, \mathrm{M}$, where $O_{i}$ and $\mathrm{E}$ represent respectively the graph of optical domain number $\mathrm{i}$ and the set of inter-domain links. We model an optical domain $O_{i}$ as an undirected graph $O_{i}=\left(V_{i}, E_{i}\right)$, where $V_{i}$ and $E_{i}$ represent respectively the set of nodes and the set of links of $O_{i}$. The set of nodes $V_{i}$ is the union of the internal node set $\mathrm{v}$ and border node set v'. Each link e in $E_{i}$ supports $w_{e}$ working wavelength channels. This working capacity is obtained by routing the demand matrix. We assume that the demand matrix, consisting of the number of wavelength channels to be established between each node pair, is given.

The ILP formulation determines the maximum number of working light-paths connecting any border nodes pair satisfying the constraints (4) and (5). The constraint (4) ensures that the number of light-paths crossing the link e should not exceed the number of the primary wavelengths available on this link. The constraint (5) guarantees that the primary light-paths interconnecting a border node pair are link disjoint of all light-paths connecting any other border node pair. The number of physical light-paths between two 
border nodes will define the capacity of the virtual link connecting these two nodes in the virtual topology.

The objective function is:

$$
\text { Maximize } \sum_{d \in D_{i}} \sum_{p \in P_{d}} h_{d p}
$$

Subject to:

$$
\begin{gathered}
\sum_{d \in D_{i}} \sum_{p \in P_{d}} a_{e, d, p} h_{d p} \leq w_{e} \quad \forall e \in E_{i} \\
\sum_{d \in D_{i}} Y_{e, d} \leq 1 \quad \forall e \in E_{i}, \forall d \in D_{i}
\end{gathered}
$$

Let $D_{i}$ be the set of border node pairs in the domain i. $P_{d}$ indicates the set of all paths connecting the border node pair d. $h_{d p}$ is an integer variable that indicated the number of lightpaths connecting the border node pair $\mathrm{d}$ installed on path $\mathrm{p}$.

$Y_{e, d}= \begin{cases}0 & \text { if } \sum_{p} a_{e, d, p} h_{d p}=0 ; \quad e \notin p, \forall p \in P_{d}, \forall d \in D_{i} \\ 1 \quad \text { if } \sum_{p} a_{e, d, p} h_{d p} \leq 0 ; \quad e \notin p, \forall p \in P_{d}, \forall d \in D_{i}\end{cases}$

$Y_{e, d}$ is a binary variable that indicates whether one of the lightpaths connecting the border node pair $d$ crosses the link e.

$$
a_{e, d, p}= \begin{cases}0 & \text { ife } \notin p, \forall p \in P_{d}, \forall d \in D_{i} \\ 1 & \text { ife } \notin p, \forall p \in P_{d}, \forall d \in D_{i}\end{cases}
$$

$a_{e, d, p}$ is a binary constant which indicates if the lightpath $\mathrm{p}$ of the border node pair $\mathrm{d}$ crosses the link e.

\section{RESUlts AND ANALYSIS}

The performances of our solution are evaluated in this section. Simulation experiments are carried out using three widely used topologies respectively taken from [7] and [16] and characterized in Tabl e1. The first two topologies contain three different domains, while the last one contains four domains. Between two different domains, the node-pair is interconnected by a bi-directional fiber link. Each fiber link is assumed to have 128 wavelengths, and each network node is assumed to have the wavelength conversion capacity. All simulations are running on DELL Quadri Dual Core Xeon processor and $4 \mathrm{~GB}$ of RAM. MATLAB is used to solve the ILP formulations.

The traffic demand is uniformly distributed among all source-destination pairs. Each demand requests for one unit of capacity. The working capacities on the network links are obtained by routing each demand over the shortest path. Several experiments are performed for each topology network and the average values are presented as the final results.

The performance metrics that we have considered are:

Redundancy: Redundancy constitutes a major evaluation criterion for WDM network design. It gives an insight on the quality and the relative cost of network protection. The redundancy enables to measure the protection efficiency:
Table I

TOPOLOGY CHARACTERISTICS

\begin{tabular}{llll}
\hline Characteristics $\backslash$ Topologies & US network & ARPANET & T3 \\
\hline Nodes & 15 & 20 & 44 \\
Links & 25 & 32 & 69 \\
Nodal degree & 3.33 & 3.2 & 3.13 \\
Demands & 128 & 174 & 490 \\
Working capacity & 245 & 457 & 1941 \\
\hline
\end{tabular}

it denotes the ratio between the spare capacity (protection capacity) and the working capacity in the network.

Computational complexity: Computational complexity is an important factor when evaluating an algorithm. It is defined as the time it takes for an algorithm to find a solution. If an algorithm can achieve a near optimal solution with an acceptable computational complexity, it will likely be a desirable solution to the problem.

Several scenarios are also considered to evaluate our solution:

Dedicated-backup: In the first scenario, we implement the dedicated 1+1 backup path protection. In dedicated backup path protection, both the working and the backup path are link disjoint. The resources along a backup path are uniquely reserved for only one working path. The complete topology of the multi-domain networks is available for the calculation of the set of p-cycles.

Full-information: In this scenario, the p-cycle based protection is implemented. The complete topology of the multi-domain networks is available for the calculation of the set of p-cycles. The p-cycles protecting the multi-domain networks are computed using the exact solution proposed in [7]. This solution uses an ILP to find the optimal set of p-cycles in terms of redundancy.

Aggregated-topology: In the third scenario, the topology of the multi-domain networks is transformed into a virtual topology by using our aggregation model. The set of pcycles is computed on the virtual topology by using the same algorithm used in the previous scenario.

Note: The working capacity remaining in each domain after the aggregation of the original topology is also protected by using p-cycles.

Single-node: In this scenario, we implement the solution proposed in [9] and described in the second section of this paper. In this solution each domain is represented by a single node and the inter-domain and intra-domain links are independently protected using p-cycles.

The results summarized in Table 2, show the values of redundancy obtained in the different scenarios. Table 3 compares the running time taken by the different scenarios to compute the set of p-cycles protecting the network.

We can see in Table 2 that the value of redundancy obtained in the second scenario Full-information is better than that obtained by the others scenarios for the first 
Table II

REDUNDANCY

\begin{tabular}{llll}
\hline Solutions $\backslash$ Topologies & US network & ARPANET & T3 \\
\hline Dedicated-backup & $149 \%$ & $153 \%$ & $138.82 \%$ \\
Full-information & $93,65 \%$ & $92,14 \%$ & - \\
Aggregated-topology & $99,19 \%$ & $96,35 \%$ & $103.78 \%$ \\
Single-node & $146,86 \%$ & $165 \%$ & $150.88 \%$ \\
\hline
\end{tabular}

two topologies. This remark is justified by the fact that when the complete topology of multi-domain networks is considered during the p-cycle computation, the resource optimization works obviously better. However, the running time of this solution is very long and grows with the size of the network as shown in the table 3 . We also note that the Full-information solution cannot find a result for the third topology. This is due to the large size of this topology and the large number of working wavelengths in the network. This was part of our motivation to propose a new topology aggregation model and adapt it to the p-cycles in order to reduce the topology complexity. Consequently, the running time taken by our solution Aggregated-topology is very short compared to that taken by the optimal Full-information solution.

The redundancy of our solution Aggregated-topology is $5.54 \%$ and $4.21 \%$ higher than the redundancy of the Fullinformation solution, respectively for the US and ARPANET test networks. The main reason for that behavior is that the working capacity remaining after the aggregation (cf. Figure $6(d))$ is protected independently of the aggregated working capacity.

Table III

COMPUTATIONAL COMPLEXITY

\begin{tabular}{llll}
\hline Solutions $\backslash$ Topologies & US network & ARPANET & T3 \\
\hline Dedicated-backup & $1.25(\mathrm{~s})$ & $2.04(\mathrm{~s})$ & $13.04(\mathrm{~s})$ \\
Full-information & $330648.09(\mathrm{~s})$ & $1055633.75(\mathrm{~s})$ & - \\
Aggregated-topology & $10.5(\mathrm{~s})$ & $15.5(\mathrm{~s})$ & $29.76(\mathrm{~s})$ \\
Single-node & $1,43(\mathrm{~s})$ & $4.56(\mathrm{~s})$ & $8.68(\mathrm{~s})$ \\
\hline
\end{tabular}

We can see also that the state-of-the-art solution Singlenode requires more resources than our solution to protect the working capacity. This difference is due to two factors:

1. During the calculation of p-cycles, our solution uses more information about the physical topology of each domain (this information comes from the virtual aggregated topologies).

2. In our solution each calculated p-cycle might protect some working capacities in the traversed domains. This is not the case for the single-node proposal. Thus, we significantly improved the redundancy value for our proposed solution.

\section{CONCLUSION}

In this article, we addressed the protection of multidomain optical networks. We started by classifying the proposed solutions and by discussing their drawbacks. we proposed a new solution for the survivability of multidomain optical networks based on p-cycles. For scalability and security reasons, we also proposed a new topology aggregation model adapted to the p-cycle computation. This aggregation model enabled our solution to improve the quality of the computed p-cycles.

Moreover, our solution protects primary light-paths within each domain using the spare capacity reserved for the existing p-cycles protecting the inter-domain links. Thus it improves significantly the redundancy parameter.

Simulation results show that our solution is a good tradeoff between two competing goals: efficient use of backup resources and small running time. This study is very encouraging, and we intend to continue our investigations by using other constraints such as the size of p-cycles and integrating the domain management policies.

\section{REFERENCES}

[1] A. Akyamac, S. Sengupta, J. Labourdette, S. Chaudhuri, and S. French, Reliability in Single domain vs. Multi domain Optical Mesh Networks, National Fiber Optic Engineers Conference, 2002.

[2] D. Truon and B. Thiongane, Dynamic routing for shared path protection in multidomain optical mesh networks. Journal of Optical Networking, 58-74, 2006.

[3] Q. Liu, M. Ko, N. Ghani and A. Gumaste, Hierarchical routing in multi-domain optical networks. Computer Communications, 122-131, 2006.

[4] D. Larrabeiti, R. Romeral, I. Soto, M. Uruena, T. Cinkler, J. Szigeti and J. Tapolcai, Multi-domain issues of resilience, in: J. Pareta (Ed.), . Int. Conf. Transparent Optical Networks, Barcelona, 375-380, 2005.

[5] M. Herzberg, F. Shleifer, R. Ring, and O. Zolberg, Applying OD Cycles to Multi-domain, Multi-service Survivable Networks, in 3rd EuroNGI Conference on the Next Generation Internet Networks, 80-87, 2007.

[6] X. Xie, W. Sun, W. Hu and J. Wang, A shared sub-path protection strategy in multi-domain optical networks, Optical Fiber Communication and Optoelectronics Conference, 2007.

[7] L. Guo, LSSP: A novel local segment-shared protection for multi-domain optical mesh networks. Computer Communications, 1794-1801, 2007.

[8] A. D'Achille, M. Listanti, U. Monaco, F. Ricciato, and V. Sharma, Diverse Inter-Region Path Setup/Establishment, IETF Internet-Draft, draft dachille diverse interregion path setup, 2004.

[9] J. Szigeti, R. Romeral, T. Cinkler and D. Larrabeiti, p-Cycle Protection in Multi-Domain Optical Networks, Springer Journal of Photonic Network Communications, 2009. 
[10] C. Huang and D. Messier, A Fast and scalable Inter-Domain MPLS Protection Mechanism, Journal of Communications and Networks, 60-67, 2004.

[11] W.D. Grover and D. Stamatelakis, Cycle Oriented Distributed Preconfiguration: Ring-like Speed with Mesh-like Capacity for Self-planning Network Restoration, International Conference on Communications, 537-543, 1998.

[12] B. Awaebuch and Y. Shavitt, Topology aggregation for directed graphs. IEEE Symposium on Computers and Communications, 1998.

[13] W. Lee, Minimum equivalent subspanner algorithms for topology aggregation in ATM networks, International Conference on ATM, 1999.

[14] K.R. Bhutani, A. Battou, and B. Khan, Two Approaches for Aggregation of Peer Group Topology in Hierarchical PNNI Networks, Int. J. Intell. Autom. Soft Comput, 2000.

[15] H. Drid, B. Cousin, S. Lahoud and M. Molnr, Multi-criteria p-cycle network design, IEEE Conference on Local Computer Networks, 2008.

[16] X. Yang and B. Ramamurthy, Inter-domain dynamic routing in multi-layer optical transport networks. IEEE GLOBECOM, 2003. 